\title{
Research on Dynamic Equivalent for Hydropower Generator Group Based on Phasor Measurement Information Part I:Theory and Method
}

\author{
Huabo SHI ${ }^{1, a}$, Gang CHEN ${ }^{1, b}$, Lijie DING ${ }^{1, c}$, Hua ZHANG ${ }^{1, d}$ \\ ${ }^{1}$ State grid Sichuan Electric Power Research Institute, Chengdu, 610072, China \\ aemail:shbo87@163.com, bemail:gangchen08@gmail.com, cemail:ding_lijie@163.com \\ demai:zhanghua002@163.com
}

Keywords: PMU; hydropower generator group; dynamic equivalent; parameter generalization

\begin{abstract}
In order to simulate the dynamic characteristics of small hydropower group more accurately, a method of dynamic equivalent for hydropower generator group use phasor measurement information is proposed. Using the idea of weighted aggregation, aggregate parameters identified in different temporal and spatial distribution into equivalent parameters of the hydropower generator group, which can solve the problems like multiple solutions due to the intelligent optimization algorithm and the equivalent parameters generalization. Equivalent method and parameters generalization method are verified in this paper.
\end{abstract}

\section{Introduction}

At the time of large-scale power system simulation, the accuracy of the generator parameters are very important to the power system security and stability. There are large amounts of hydropower generators in southwest region, it's so difficult to measure all generators parameters and modeling, usually the small hydropower generators are unmodeled, which will seriously affect the accuracy of the simulation. For example, at the time of security and stability analysis of provincial power grid, the small hydropower generator group is Replaced with negative load, which can make the simulation results deviation larger and bring hidden trouble to the safety of the power grid operation.

Dynamic equivalence is one of the most traditional research direction of power system, a large amount of research on the system dynamic equivalent model and parameter identification method are studied by many scholars and achieved abundant research results. Reference 1 researched on a hybrid dynamic equivalent using ANN-based boundary matching technique;

Reference 2 proposed a model based on grey box and equivalence method use artificial neural network; Reference 3 used structure preservation technique as dynamic equivalent modeling method; coherency based equivalence method is used in Reference 4- Reference 6; equivalent method provided by NETOMAC software is used in Reference 7.

Looking from the existing Reference, most researched the equivalent based on the power system known model and parameters. There is little Reference studies on equivalent parameter generalization and adaptation, which Has a negative effect on equivalent method applied in the actual power grid.

Therefore, this paper researches on dynamic equivalent for hydropower generator group based on phasor measurement information of tie-line. Based on the idea of weighted aggregation, aggregate parameters identified in different temporal and spatial distribution into equivalent parameters of the hydropower generator group. Equivalent method and parameters generalization method are verified in the paper.

\section{The dynamic equivalent model and parameter identification method}

\section{A. Dynamic equivalent model}

Hydropower sending base, generally, multiple plants work in parallel and send out power by tie-line which is radial distribution grid. The power grid with low voltage parallel in the 
hydropower channel, the electromagnetic loop network is decoupled. This kind of power grid, under the condition of power grid structure, the component data are unknown, the small generators can approximately considered as coherent. Therefore, the above system can be equivalent as a generator parallel with load shown in figure 1.

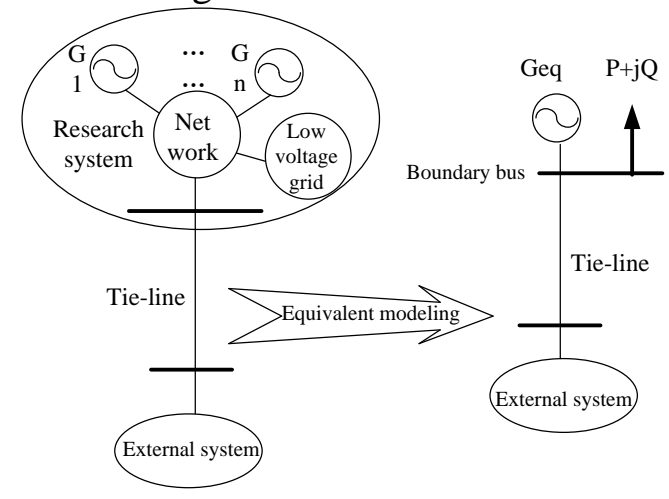

Fig. 1. Single-line contact region dynamic equivalence schematic

\section{B. Equivalent generator and load model}

generator model takes frequency as the input signal shown in type (1), electromagnetic power and load shown in type (2) and type (3).

$$
\begin{aligned}
& T_{j} \frac{d \omega}{d t}=P_{m}-P_{e}-D \frac{d \delta}{d t} \\
& \frac{d \delta}{d t}=\left(\omega-\omega_{f}\right) \omega_{0} \\
& T_{d}^{\prime} \frac{d E^{\prime}}{d t}=E_{f 0}-K_{V}\left(V-V_{0}\right)-\frac{x_{d}}{x_{d}^{\prime}} E^{\prime}+\left(\frac{X_{d}}{x_{d}^{\prime}}-1\right) V \cos (\delta) \\
& P_{e}=\frac{E^{\prime} V}{x_{d}^{\prime}} \sin (\delta) \\
& Q_{e}=\frac{E^{\prime} V \cos (\delta)-V^{2}}{x_{d}^{\prime}} \\
& P_{s}=P_{s 0}\left(V / V_{0}\right)^{2} \\
& Q_{s}=Q_{s 0}\left(V / V_{0}\right)^{2}
\end{aligned}
$$

The tie line power equation is:

$$
\left\{\begin{array}{l}
P_{l}=P_{e}-P_{s} \\
Q_{l}=Q_{e}-Q_{s}
\end{array}\right.
$$

Where, $T_{j}$ is inertia time constant; $P_{m}$ is mechanical power; $E^{\prime}$ is potential back of $x_{d}^{\prime}$; $\mathrm{V}$ is generator voltage, $\mathrm{V} 0$ is the initial voltage of the machine; $x_{d}$ is transient reactance of $\mathrm{d}$ axis, $x_{d}$ is synchronous reactance of $\mathrm{d}$ axis; $\delta$ is the angle between the voltage and the electric potential; $D$ is damping coefficient; $\omega$ is rotor speed, $\omega_{f}$ is bus frequency; $T_{d o}$ is $\mathrm{d}$ axis transient open circuit time constant; $E_{f 0}$ is initial excitation voltage; $K_{v}$ is the excitation voltage feedback coefficient; $P_{s 0} 、 Q_{s 0}$ is load base value of active and reactive power respectively; $P_{l} 、 Q_{l}$ is active and reactive power on tie-line respectively. Type (1) - (4) constitute dynamic equivalence model of small hydropower group.

\section{Equivalent parameter identification}

Four input variables of equivalent model are the actual voltage, frequency, power of tie-line respectively $\left[V_{\text {real }} 、 \omega_{\text {real }} 、 P_{\text {lreal }} 、 Q_{\text {lreal }}\right]$, output variables are active power and reactive power identified $\left[P_{l} 、 Q_{l}\right]$, eight parameters to be identified are $\left[x_{d} 、 x_{d}, T_{d 0}, T_{j}, D 、 K_{v} 、 P_{s 0} 、 Q_{s 0}\right]$.

Injecting the voltage, frequency, power signal of tie-line after disturbance occurs in external power grid into the equivalent model, which will produce equivalent active power and reactive power of tie-line $P_{l} 、 Q_{l}$. Combined with the tie line power oscillation waveform, deviation function 
defined as:

$\left\{\begin{array}{l}e=\sum_{i=1}^{i \leq n} \sqrt{A(i)^{2}+B(i)^{2}} \\ A(i)=P_{l}(i)-P_{\text {Ireal }}(i) \\ B(i)=Q_{l}(i)-Q_{\text {Ireal }}(i)\end{array}\right.$

Where, $n$ is sampling points of oscillation waveform data. Using the improved particle swarm optimization (pso) algorithm to search parameters, when the deviation convergence to minimum, equivalent system parameters is identified. Parameter identification process shown in figure 2.

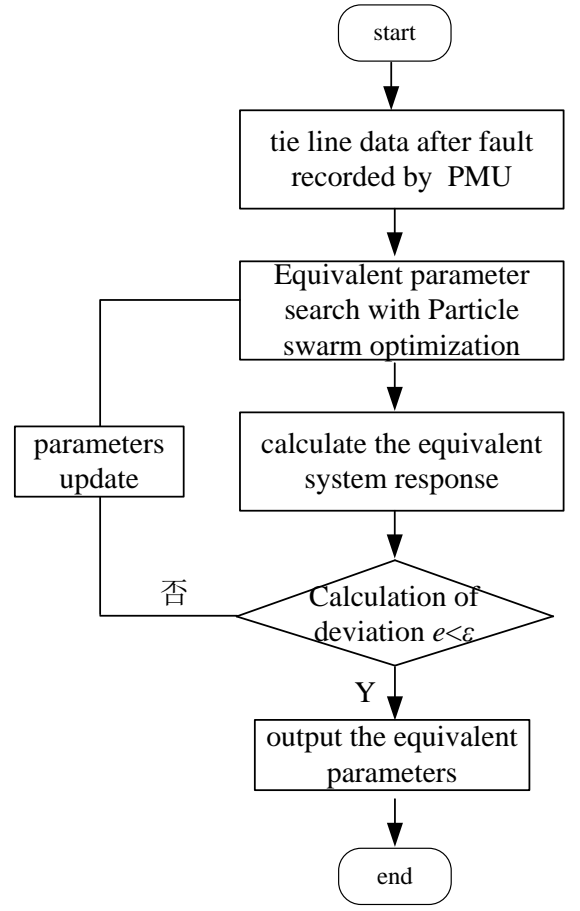

Fig. 2. Equivalent system parameter identification process

\section{Equivalent parameters generalization}

There will be problem as multiple solution use intelligent algorithm to identify parameter inevitably. We will get a different set of parameters for each evaluation. In addition, even if the system runs in the same way, system response caused by disturbance In different time-space distribution is different. The equivalent model and parameters only for a particular response under the disturbance is strict, but unable to describe other disturbances accurately.

Therefore, this paper presents a parameter generalization method based on the weighted average aggregation method. The basic idea is: Using a disturbance data may get multiple sets of equivalent parameters, different disturbance data can get different equivalent parameters, and then weighted average method is used to aggregate final parameters like inertial time constant and damping coefficient, etc. This method can solve the multiple solution problem caused by intelligent optimization algorithm and generalization, adaptation of the equivalent parameters. Define solving formula as follows:

$$
\left\{\begin{array}{l}
X_{f h}=\frac{\sum_{i=1}^{N} X_{e q . i}}{N} \\
X_{e q . i}=\frac{\sum_{j=1}^{M} X_{j}}{M}
\end{array}\right.
$$

Where, $X_{f h}$ is system equivalent aggregation parameters; $i$ is the disturbance number; $N$ is the total number of disturbance; $X_{\text {eqi }}$ is the equivalent parameter identified under the $i$-th disturbance; $X_{j}$ is the result calculated in $j$-th under one disturbance; $j$ is the optimization calculation number 
under one disturbance; $M$ is total number of calculation in one disturbance.

\section{Analysis of example}

\section{A. Dynamic equivalent method verification}

Using a generator and parallel load link to Sichuan power system as model studied, the equivalent parameters identified shown in table 1. Response under same fault before and after equivalent shown in figure 3.

Table 1 Equivalent parameters identified

\begin{tabular}{ccccccccc}
\hline & $T_{j}$ & $x_{d}{ }^{\prime}$ & $K_{v}$ & $T_{d 0}{ }^{\prime}$ & $x_{d}$ & $D$ & $P_{s 0}$ & $Q_{s 0}$ \\
\hline Real value & 53.613 & 0.0523 & $/$ & 13.475 & 0.1627 & 0.2 & 1 & 0.5 \\
identified & 54.827 & 0.0501 & 2.5148 & 9.8935 & 0.2906 & 0.3029 & 0.9668 & 0.1084 \\
error & $2.26 \%$ & $-4.21 \%$ & $/$ & $-26.57 \%$ & $78.61 \%$ & $51.45 \%$ & $-3.32 \%$ & $-78.32 \%$ \\
\hline
\end{tabular}

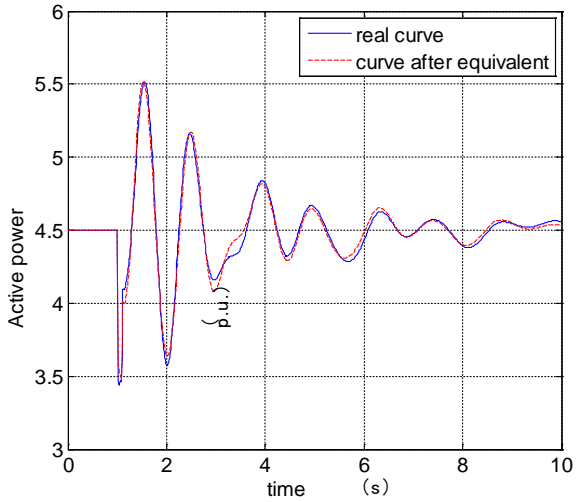

(a)Active power response

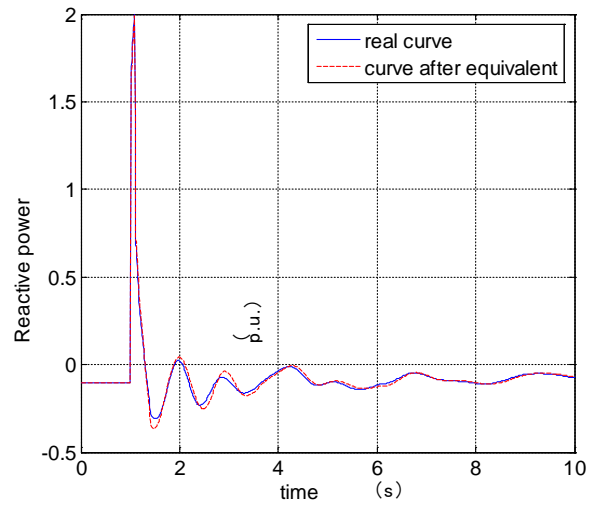

(b)Reactive power response

Fig. 3. Equivalent system parameter identification process

According to the calculation results, inertial time constant, the transient reactance and active load is consistent, the error is less than $\pm 5 \%$. Due to other parameters influence on the system dynamic response is relatively small, which can't identified accurately. Besides, generator model to be identified is different with the equivalent model(Excitation system), the parameters identified should different with the real value. The active power and reactive power response before and after equivalent is consistent.

\section{B. Equivalent parameter generalization method verification}

Because the accurate equivalent parameters of hydropower channel are unknown, unable to determine the equivalent accuracy directly. Defining the similarity between the real response and the equivalent response under the same 验证 disturbance is:

$\rho_{(\text {preal }, p l)}=\frac{\operatorname{Cov}\left(P_{\text {Ireal }}, P_{l}\right)}{\sqrt{D\left(P_{\text {lreal }}\right)} \sqrt{D\left(p_{l}\right)}}$

$\begin{cases}0.8 \leq\left|\rho_{\left(P_{\text {real }}, P_{1}\right)}\right| \leq 1 & \text { Highly similar } \\ 0.5 \leq\left|\rho_{\left(P_{\text {real }}, P_{1}\right)}\right| \leq 0.8 & \text { Re markably similar }\end{cases}$

Where, $\operatorname{Cov}\left(P_{\text {lreal }} P_{l}\right)$ is covariance of the actual power curve and equivalent power system response curve; $D\left(P_{\text {lreal }}\right) 、 D\left(P_{l}\right)$ is the variance of the actual power curve and equivalent power system response curve. Similarity calculation should consist of steady state and transient state process. This similarity index is used to evaluate the accuracy of equivalent model and effectiveness of the parameter generalization method .

Using parameter aggregation equivalent method proposed above, identify the equivalent system model parameters with response of a hydropower channel in western Sichuan power grid under five different disturbances, take a weighted average of each parameter and aggregate a set of parameters, then the adaptive capacity of the aggregated parameters under the five known disturbances is verified. The calculation results are shown in table 2. 


\section{1) Adaptability for known fault}

The similarity between the response of the aggregated parameters under the five known disturbances and the original system response curve shown in figure 4(a). From the calculation result, the active response of the aggregated system is similar to the original system highly, reactive response is remarkably similar. It turned out that the adaptability of the aggregated system is good under the five known disturbances.

Table 2.Equivalent parameters aggregated with five known disturbances

\begin{tabular}{ccccccccc}
\hline The disturbance number & $T_{j}$ & $x_{d}{ }^{\prime}$ & $K_{v}$ & $T_{d 0}{ }^{\prime}$ & $x_{d}$ & $D$ & $P_{s 0}$ & $Q_{s 0}$ \\
\hline 1 & 87.5414 & 0.0409 & 30.0000 & 13.5426 & 1.1243 & 0.5843 & 0.0753 & 0.3161 \\
2 & 87.8571 & 0.0410 & 51.6307 & 10.0000 & 1.5371 & 0.6129 & 0.0442 & 0.3380 \\
3 & 84.6377 & 0.0362 & 26.8819 & 21.8011 & 1.2071 & 1.0099 & 0.3873 & 0.4993 \\
4 & 86.1244 & 0.0354 & 52.2258 & 20.2443 & 1.5391 & 1.1604 & 0.1000 & 0.3198 \\
5 & 78.0393 & 0.0471 & 32.2688 & 14.8746 & 1.1828 & 0.4890 & 0.0672 & 0.3153 \\
Aggregated parameters & 84.8400 & 0.0401 & 38.6014 & 16.0925 & 1.3181 & 0.7713 & 0.1348 & 0.3577 \\
\hline
\end{tabular}

\section{2) Generalization and explanation ability to the unknown fault}

Using the other five disturbance not involved in the aggregation to verify to generalization and explanation ability to the unknown fault. The similarity between the response of the aggregated parameters under the five disturbances not involved in the aggregation and the original system response curve shown in figure 4(b).

From the calculation result, the adaptability of the aggregated system is good under the other five known disturbances not involved in the aggregation, the similarity of active power is better than the reactive power. The equivalent model is mainly used for electromechanical transient analysis, the relationship between active power is bigger. So, small reactive power deviation dose less impact on the analysis results.

The equivalent system model based on parameter aggregation simplifies the system, but it can reflect the main dynamic characteristics after disturbances. So, the equivalent model by weighted aggregation from different disturbances has certain ability to describe the known disturbances and other faults not involved in the aggregation. That is, the equivalent model and parameters have certain generalization ability.
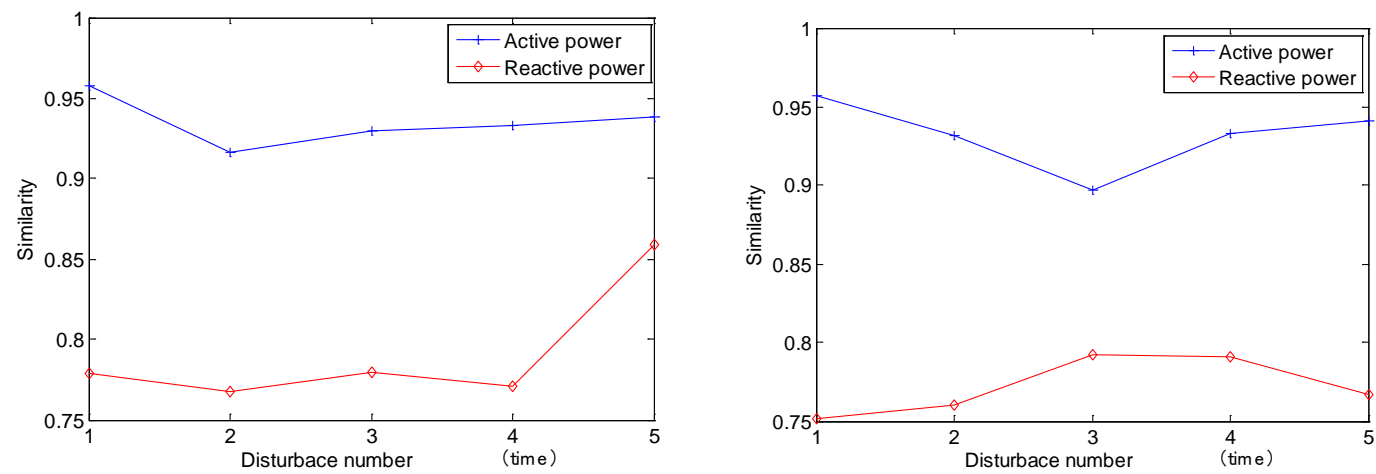

(a) under the five known disturbances

(b) under disturbances not involved in aggregation

Fig.4. The similarity between the response of equivalent and original system

\section{Conclusion}

The dynamic equivalent method for hydropower generator group based on phasor measurement information use 3 order generator and parallel load model requires less input data, equivalent accuracy is high.

Using the idea of weighted aggregation, aggregate parameters identified in different temporal and spatial distribution into equivalent parameters of the hydropower generator group, which can solve the problems like multiple solutions due to the intelligent optimization algorithm and the equivalent parameters generalization and it's suitability. That can simulate the dynamic characteristics of small hydropower group more accurately. 


\section{References}

[1]Feng Ma, Vijay Vittal.A Hybrid Dynamic Equivalent Using ANN-Based Boundary Matching Technique[J], IEEE TRANSACTIONS ON POWER SYSTEMS, 2012, 27(3): 1494 - 1502.

[2]Aleksandar M. Stankovic', Andrija T. Saric'. Transient Power System Analysis With Measurement-Based Gray Box and Hybrid Dynamic Equivalents[J]. IEEE TRANSACTIONS ON

POWER SYSTEMS, 2004, 19(1): 455 - 462.

[3]M. Larbi Ourari , Louis-A. Dessaint, Van-Que Do, IEEE Dynamic Equivalent Modeling of Large Power Systems Using Structure Preservation Technique[J], IEEE TRANSACTIONS ON POWER SYSTEMS, 2006,21(3):1284-1295.

[4]Liu Y, Li X, Wang Y, et al. Research on small signal stability of power system with distributed small hydropower[C]. Innovative Smart Grid Technologies-asia, IEEE, 2012:1-6.

[5]ZHANG Yuan-sheng. The effects on stable running of power grid of the distributed network with small hydropower[D]. Hunan University, 2012.

[6]YANG Jing-ping,XU Zheng. APPLICATION OF DYNAMIC EQUIVALENCE BASED ON IDENTIFICATION OF COHERENT GENERATOR GROUP IN ENGINEERING[J].Power System Technology,2005,29(17):68-71.

[7] Kang Yi,Zhou Xianlin,Xie Guo'en.USING NETOMAC PROGRAM IN SYSTEM EQUIVALENT STUDY[J]. Power System Technology, 1998, 22(5):21-24. 